\title{
Tecnologia de Sensoriamento 3D Hall aplicada em Sistema Eletrônico de Comando Automatizado da Transmissão É UMA SoluÇÃo que Coloca o Brasil na Vanguarda TeCnOlógica
}

\author{
Rodrigo Kraft Florêncio ${ }^{1}$, Fabio Eduardo Gennaro ${ }^{1.2}$, Anderson Moraes Amorim ${ }^{1.3}$, \\ Wilson Nogueira da Silva ${ }^{2}$ \\ ${ }^{1}$ Magneti Marelli \\ ${ }^{2}$ TE Connectivity
}

Emails: rodrigo.florencio@magnetimarelli.com, fabio.gennaro@magnetimarelli.com, anderson.amorim@magnetimarelli.com, wsilva@te.com.

\section{RESUMO}

Os grandes aglomerados urbanos brasileiros amargam congestionamentos cada vez maiores. De acordo com dados do Departamento Nacional de Trânsito (Denatran), circulam pelas ruas e estradas do país cerca de 45 milhões de veículos. A região Sudeste concentra 24 milhões de veículos, e desses, 6 milhões transitam na cidade de São Paulo. Além da capital paulista, Rio de Janeiro, Brasília e Recife são algumas das capitais que não suportam mais os freqüentes engarrafamentos.

Segundo o Instituto de Estudos Avançados da USP a soma do tempo perdido pelas pessoas no trânsito atinge a média de 240.000 horas por dia, essa quantidade de horas influência de forma direta a saúde da população, seja em função do stress gerado ou por decorrência da poluição, pois todas as pessoas expostas à rotina nas ruas apresentaram substâncias tóxicas no organismo e chance dobrada de desenvolver câncer do pulmão. Além disto são desperdiçados cerca de 204 milhões de litros combustível. (i.e [1])

Os impactos do trânsito citados acima por si só já justificam a necessidade de desenvolver veículos com tecnologias que auxiliem na redução de consumo de combustível, redução no nível de emissões de $\mathrm{CO} 2$ e aumento no conforto.

Somado ao impacto do trânsito a indústria automobilística nacional também necessita aumentar o conteúdo tecnológico em função da perda de mercado para os veículos importados. O governo atento para essa necessidade e com o objetivo de fomentar a tecnologia nacional lançou o regime automotivo Inovar Auto.

\section{INTRODUÇÃO}

O sistema powertrain é composto por motor e transmissão, sua função é de movimentar o veículo e tem impacto significativo quanto a nível de emissão de $\mathrm{CO}$, conforto e consumo de combustível.

A transmissão tem a responsabilidade de transferir para as rodas do veículo a energia do motor, adaptando a potência gerada pelo motor, convertendo torque e rotação com objetivo de proporcionar a melhor eficiência energética atendendo a necessidade de utilização. 
As transmissões presentes no mercado estão divididas em cinco grupos:

- Manual (MT);

- $\quad$ Automática (AT);

- Continuamente variável (CVT);

- Transmissão de Dupla Embreagem ( DCT);

- Automatizada (AMT).

No Brasil predomina-se o uso de transmissão manual em função do fator custo, porém poder descansar o pé esquerdo quando se está em congestionamentos, está mais barato graças à popularização dos câmbios automáticos e, mais recentemente dos automatizados. (i.e [2]). A oferta deste tipo de transmissão é crescente e para garantir este crescimento é importante que todos os componentes aplicados neste tipo de transmissão acompanhem a evolução tecnológica, por exemplo, o sistema de sensoriamento.

Portanto durante este artigo, será apresentada que a transmissão automatizada Free Choice $\AA^{\circledR}$ com a aplicação da tecnologia 3D Hall como sensor de posição, propícia atender os principais pilares do regime Inovar Auto:

- Aumento do conteúdo local;

- Investimento em inovação tecnológica local;

- Redução de peso do veículo;

- Aumento da eficiência energética (redução de consumo).

1. PRINCíPIOS BÁSICOS DE SISTEMA DE TRANSMISSÃO (i.e [3], [4], [5], [6])

O funcionamento do sistema powertrain pode ser observado na figura 01 , onde 0 motor gera um movimento rotativo (a) que é transferido para o disco de embreagem (b). O disco de embreagem está fisicamente ligado ao eixo de entrada da transmissão, por isso, o movimento rotativo (b) é o mesmo no ponto (c), quando este esta fisicamente acoplado ao motor

As relações de engrenagens da transmissão tem a função de adaptar a potência gerada pelo motor as rodas, convertendo torque e rotação. O movimento no eixo de saída (d) depende desta relação de engrenagens. Em um sistema de transmissão manual a escolha da relação é feita pelo motorista, enquanto que nas demais transmissões é feita de forma automática. O diferencial é responsável por mudar a direção do movimento (d) para os eixos de tração (e). 


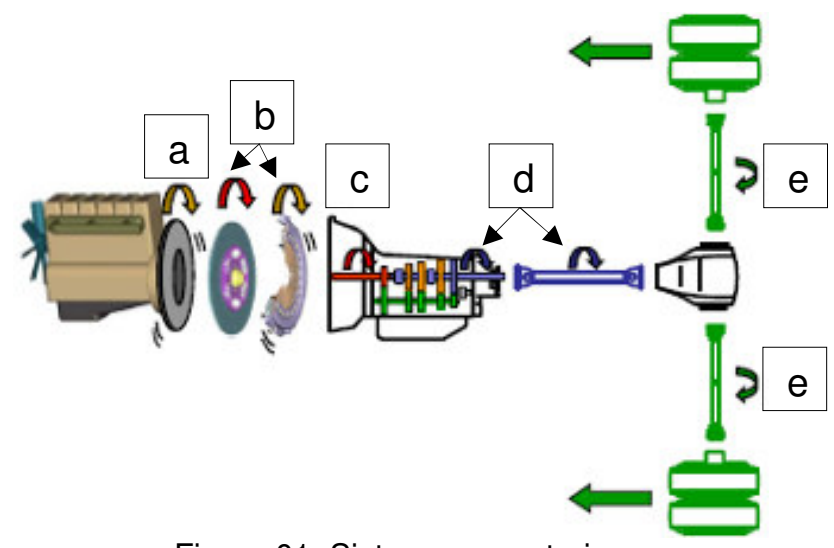

Figura 01: Sistema powertrain

\subsection{Transmissão Manual (MT)}

É o tradicional sistema que por meio da alavanca de câmbio é acionado um sistema de engrenagens de diferentes tamanhos para adequar 0 funcionamento do motor às necessidades de rotação e torque que as situações de deslocamento exigem do veículo. É o mais barato e eficiente modelo de transmissão. Sua grande desvantagem é a perda de torque durante as trocas de marcha. Em situações de tráfego intenso principalmente em congestionamentos - seu uso torna-se cansativo, além de pouco econômico. Na figura 02 demonstra o conceito de funcionamento deste tipo de transmissão.

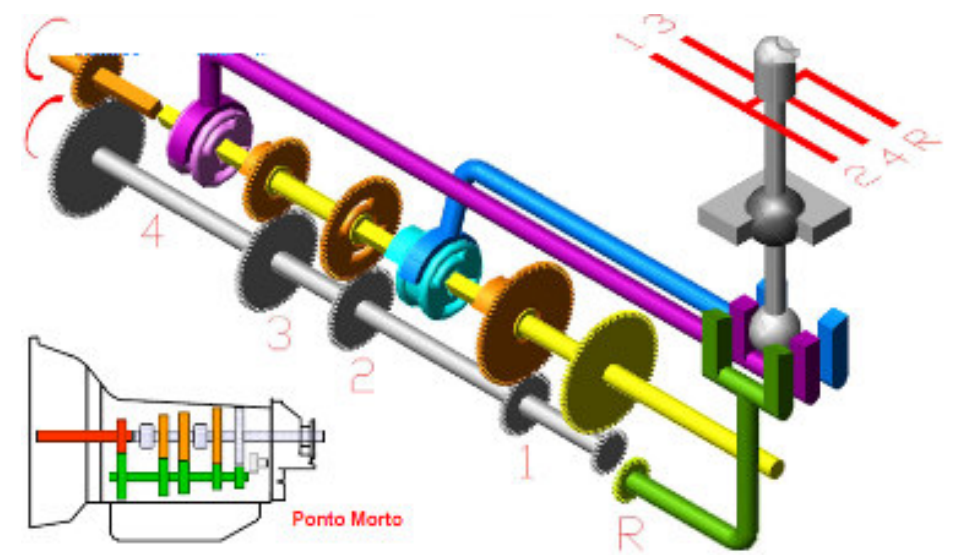

Figura 02: Esquema funcionamentotransmissão manual

\subsection{Transmissão Automática (AT)}

Esse sistema detecta a relação de velocidade e rotação do motor para realizar a troca de marcha automaticamente o que, além de um maior conforto para o motorista, mantém o motor a uma rotação quase constante, sem perda em sua capacidade de torque. Por outro lado, trata-se de um sistema maior, mais pesado, de custo elevado, com menor eficiência na transmissão e que exige mais potência do motor, ocasionando maior consumo de combustível. Dentro do grupo de transmissão automática existem diversos subgrupos (ex: sistema automático hidáulico clássico, 
sistema automático seqüencial). A figura 03 é um exemplo desta transmissão.

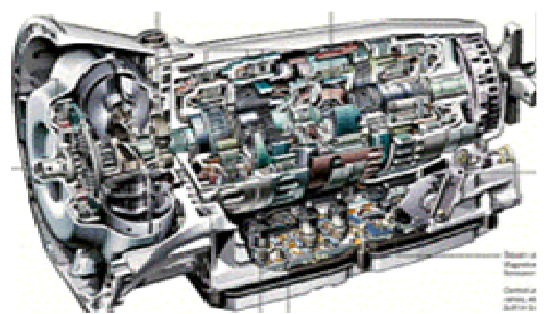

Figura 03: Exemplo de transmissão automática

\subsection{Transmissão Variável Continuada (CVT)}

Ao invés das tradicionais engrenagens, a troca de marchas ocorre por meio da ação de polias e correias ou correntes. Isso possibilita um número ilimitado de estágios para o aumento ou diminuição no torque do motor. Nesse modelo, as polias são movidas pela pressão hidráulica, força centrífuga, tensão da mola ou motores elétricos. Funciona como se houvesse infinitas marchas, o que the confere grande economia de combustível. A figura 04 é um exemplo desta transmissão.

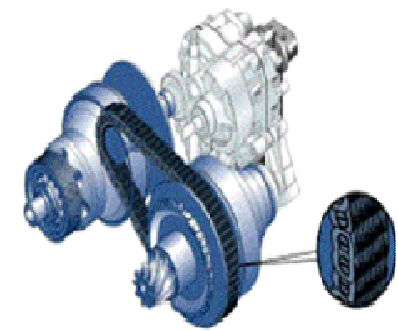

Figura 04: Exemplo de transmissão CVT

\subsection{Transmissão de Dupla Embreagem ( DCT)}

Este sistema consiste no uso de duas placas de embreagens que funcionam em conjunto como uma transmissão dentro da outra, alternadamente, por meio de engrenagens sincronizadas e mecanismo de controle eletro-hidráulico. É o sistema com maior eficiência na relação de troca de marcha, torque e velocidade, desenvolvido inicialmente para carros superesportivos. Pela sua complexidade de fabricação e manutenção, este é o sistema mais caro de todos. A figura 05 é um exemplo desta transmissão.

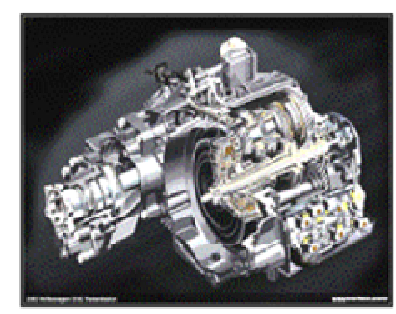

Figura 05: Exemplo de transmissão DCT 


\subsection{Transmissão Automatizada (AMT)}

A Transmissão Manual Automatizada (AMT), também conhecida como Free Choice ${ }^{\circledR}$ produzida pela Magneti Marelli, consiste em um mecanismo eletrohidráulico para automatização da transmissão manual (figura 06). Adaptada da Fórmula 1 , o sistema combina o conforto em sua utilização a uma redução no consumo de combustível e pode ser aplicada em qualquer tipo de transmissão manual, com custos de produção menores em comparação às transmissões automáticas tradicionais.

O sistema AMT se baseia em uma unidade de controle eletrônico e sistema hidráulico que permite que o motorista mude a marcha sem utilizar a embreagem, de forma sequencial ou totalmente automática.

O dispositivo da Magneti Marelli opera na transmissão manual de um carro da mesma forma que o motorista: ele abre e fecha a embreagem, engata e desengata as marchas e, quando necessário, controla também a escolha do modo de operação (automático ou sequencial para a troca de marcha). Esses três movimentos de controle são garantidos por três atuadores hidráulicos específicos, controlados pelas válvulas eletrohidráulicas.

Todos os componentes na unidade hidráulica estão reunidos em um kit único (figura 07). Ele é entregue ao fabricante do carro selado e pronto para ser instalado na transmissão. Assim que montado, o kit hidráulico mecanicamente faz interface com o eixo de acionamento da marcha.

O coração e a inteligência do sistema de controle de marcha consistem na TCU (Unidade de Controle de Transmissão). Considerando as exigências do motorista e as condições operacionais do veículo, ela gerencia as mudanças de marcha controlando a embreagem, as marchas e o motor.

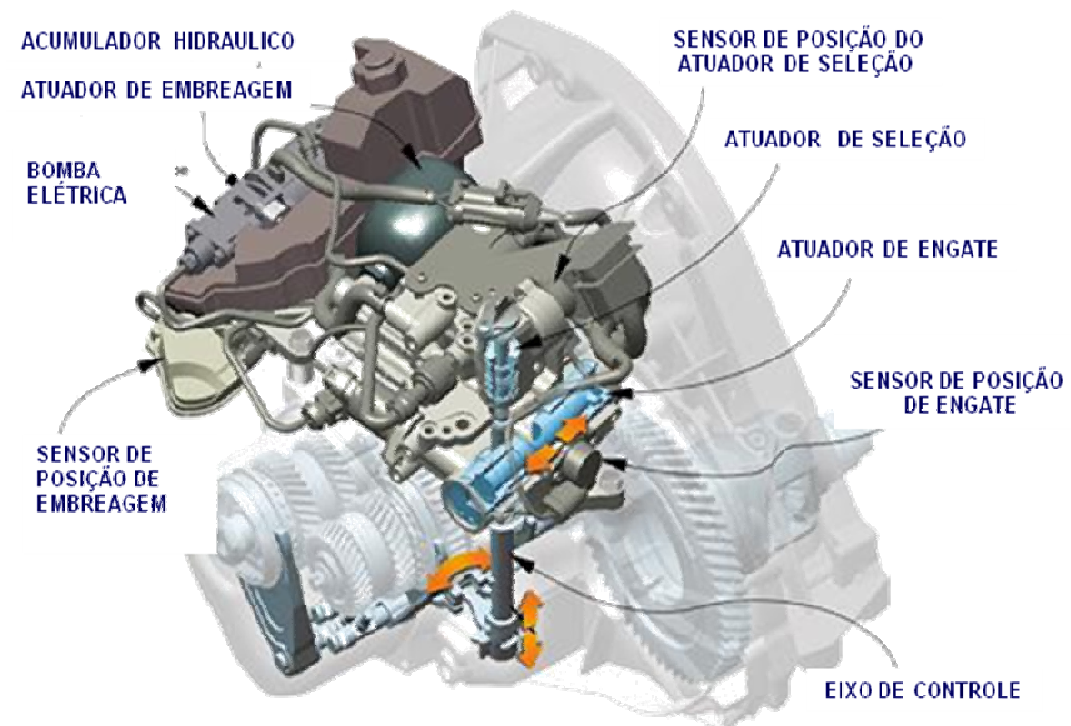

Figura 06: Exemplo de transmissão AMT 


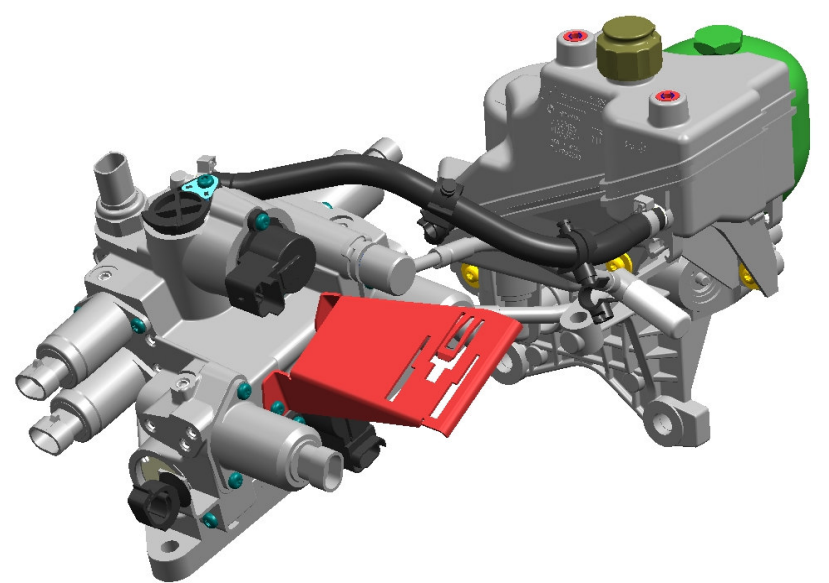

Figura 07: AMT Free Choice®

Na figura 08 é esquema hidráulico de funcionamento.

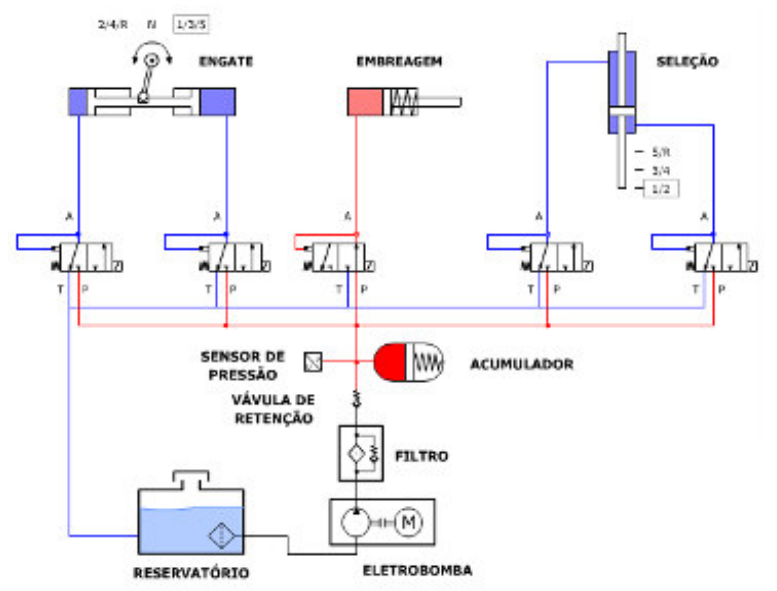

Figura 08: Esquema hidráulico

Graças à otimização eletrônica da mudança de marcha e à ligação com o controle do motor, a AMT Free Choice ${ }^{\circledR}$ consegue garantir menos consumo do que um carro com transmissão manual e a redução também significa uma redução na emissão de CO2. Em algumas das melhores aplicações da AMT em carros produzidos em escala industrial, observou-se uma redução de $5 \%$ no consumo de combustíveis e $5 \mathrm{~g} / \mathrm{km}$ nas emissões de $\mathrm{CO} 2$ em comparação a automóveis com transmissão automática. A figura 09 demonstra um comparativo.

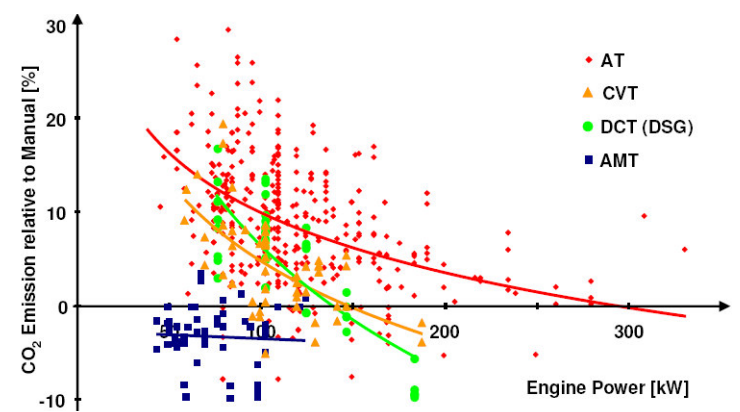

Figura 09: Comparativo emissões CO2 


\subsection{Comparativo entre os sistemas de transmissão}

Para efeito de padronização da base de comparação entres diferentes tipos de sistemas de transmissão, dividiremos os grupos acima da seguinte forma:

- Transmissão Manual de 5 Velocidades (MT5S) - referência para comparação;

- Transmissão Automática 4 Velocidades (AT4S);

- Transmissão Automática 8 Velocidades (AT8S);

- Transmissão Automatizada (AMT);

- Transmissão Dupla Embreagem (DCT).

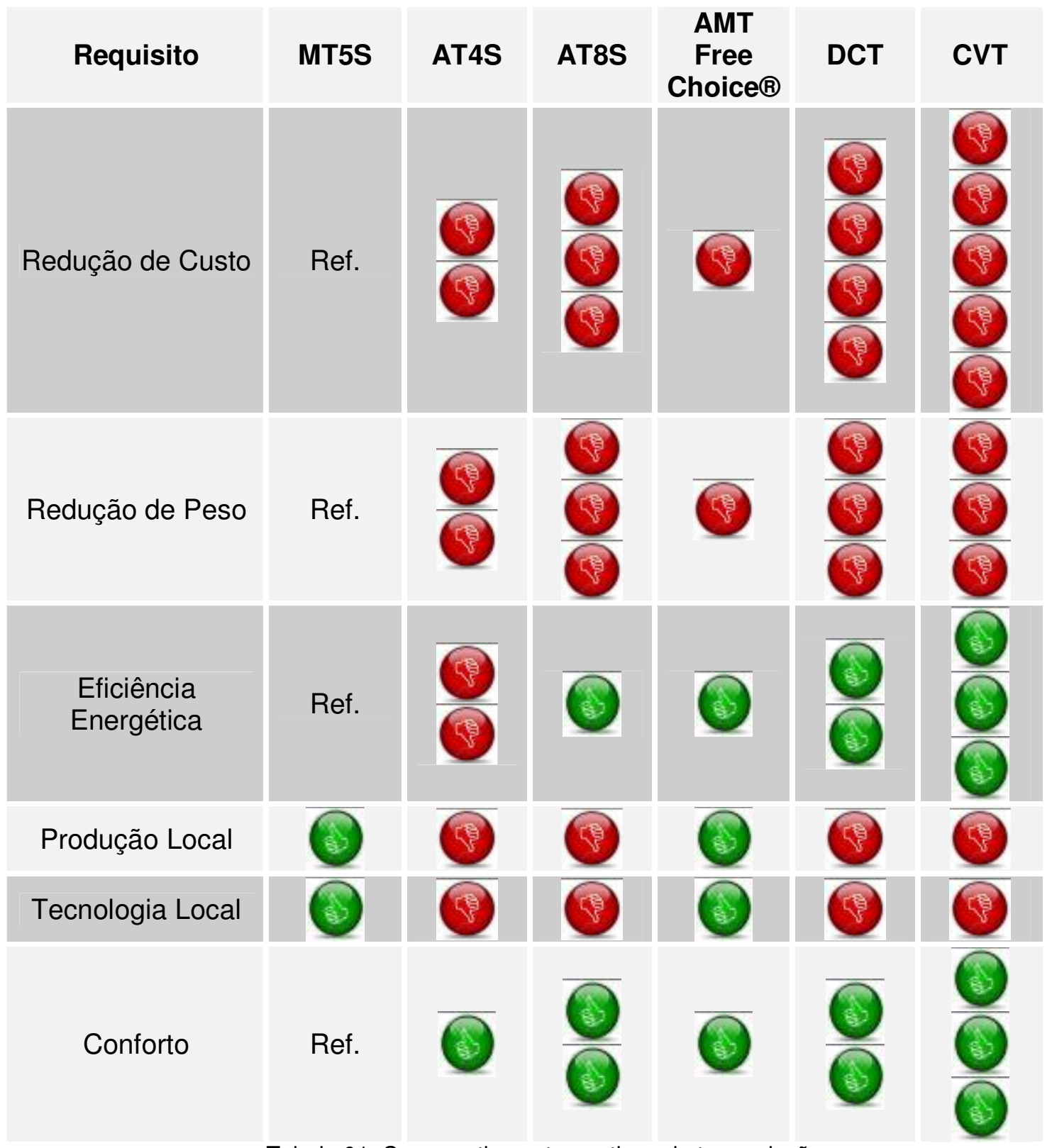

Tabela 01: Comparativo entre os tipos de transmissão 
Com base na tabela acima percebemos que dentre os sistemas comparados, AMT - Free Choice® é o que apresenta melhor eficiência com referência a transmissão manual.

\section{SISTEMA DE SENSORIAMENTO DE POSIÇÃO (I.e [7],[8])}

Inicialmente é importante dividir os tipos de sensores de posição em 3 grandes grupos:

- Sensores com Contato (Elétrico e Mecânico);

- Sensores sem Contato Elétrico, porém com contato Mecânico;

- Sensores sem Contato (full Contactless).

\subsection{Sensores com Contato (Elétrico e Mecânico) (i.e [9])}

Este tipo de sensore foi amplamente utilizado pela indústria automobilística na década de 90 em função dos benefícios de custo ao ser comparado com os custos da época para as demais tecnologias de sensoriamento de posição.

A principal tecnologia de sensor de posição com contato é a popularmente denominada potenciômetro.

Nesta tecnologia se faz necessário o contato mecânico entre o elemento móvel que precisa ser monitorado e também do contato entre as molas de contato que desliza sobre a pista de carbono e que será responsável pela resposta elétrica que define o posicionamento.

O principal problema desta tecnologia deve-se ao fato do contato gerar desgastes nos componentes, limitando substancialmente a expectativa de vida do componente. Na figura 10 pode-se observar o contato mecânico entre o elemento resistivo e o cursor.

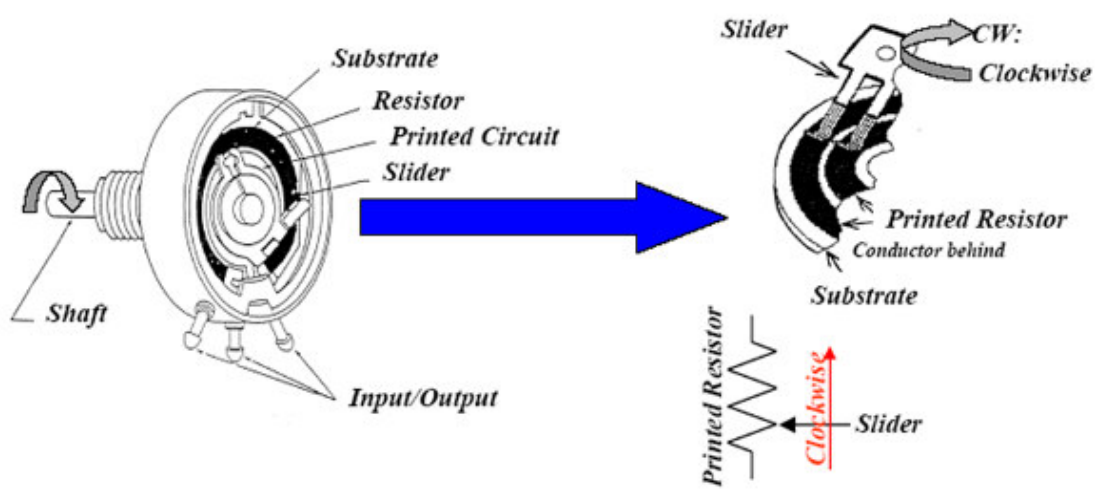

Figura 10: Esquema de funcionamento do sensor com contato

Na figura 11 são exemplos de sensores com contato. 


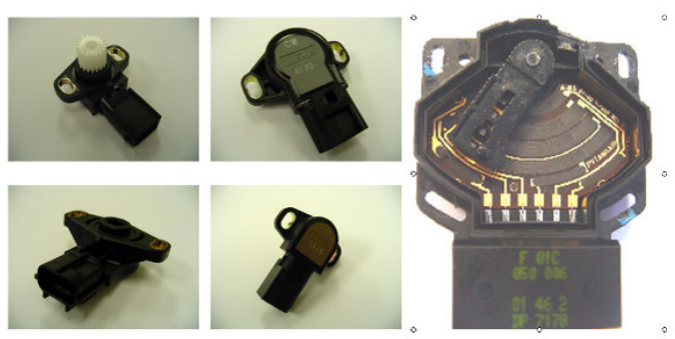

Figura 11: Exemplos de sensor com contato

\subsection{Sensores sem Contato Elétrico, porém com contato Mecânico}

Com o objetivo de elevar a expectativa de vida dos sensores de posição, a indústria automobilística passou a utilizar uma solução hibrida. Onde o contato elétrico foi substituído por tecnologias mais sofisticadas de sensoriamento (ex: Hall) porém foram mantidos os contatos mecânicos entre o elemento sensor e o componente a ser monitorado. Na figura 12 é um exemplo deste sensor.

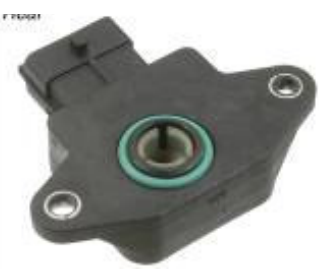

Figura 12: Exemplo de sensor sem contato elétrico

Este tipo de sensor possibilitou garantir, para uma série de aplicações, a expectativa de vida compatível com o sistema, contudo em função de permanecer o contato mecânico, o desgaste continuou sendo o fator limitante. Desta forma a aplicação continuou crítica para sistemas que demanda um número acentuado de acionamento e ou aplicações onde o posicionamento do sensor é de difícil acesso e por isto implicaria em alto custo de manutenção.

\subsection{Sensores sem contato (full contactless) (i.e[10])}

Este tipo de sensor não tem contato elétrico, nem mecânico é denominado full contactless. É atualmente o mais aplicado em novos projetos da indústria automobilística para monitoramento de posicionamento mecânico com movimento linear ou angular. A figura 13 é um exemplo de sensor full contactless.

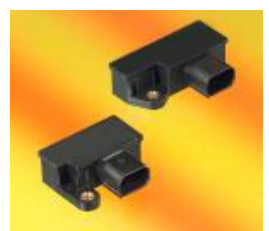

Figura 13: Exemplos de sensor full contactless 
Várias tecnologias são utilizadas para o desenvolvimento de Sensores "Full Contactless", a figura 14 demonstra algumas delas:

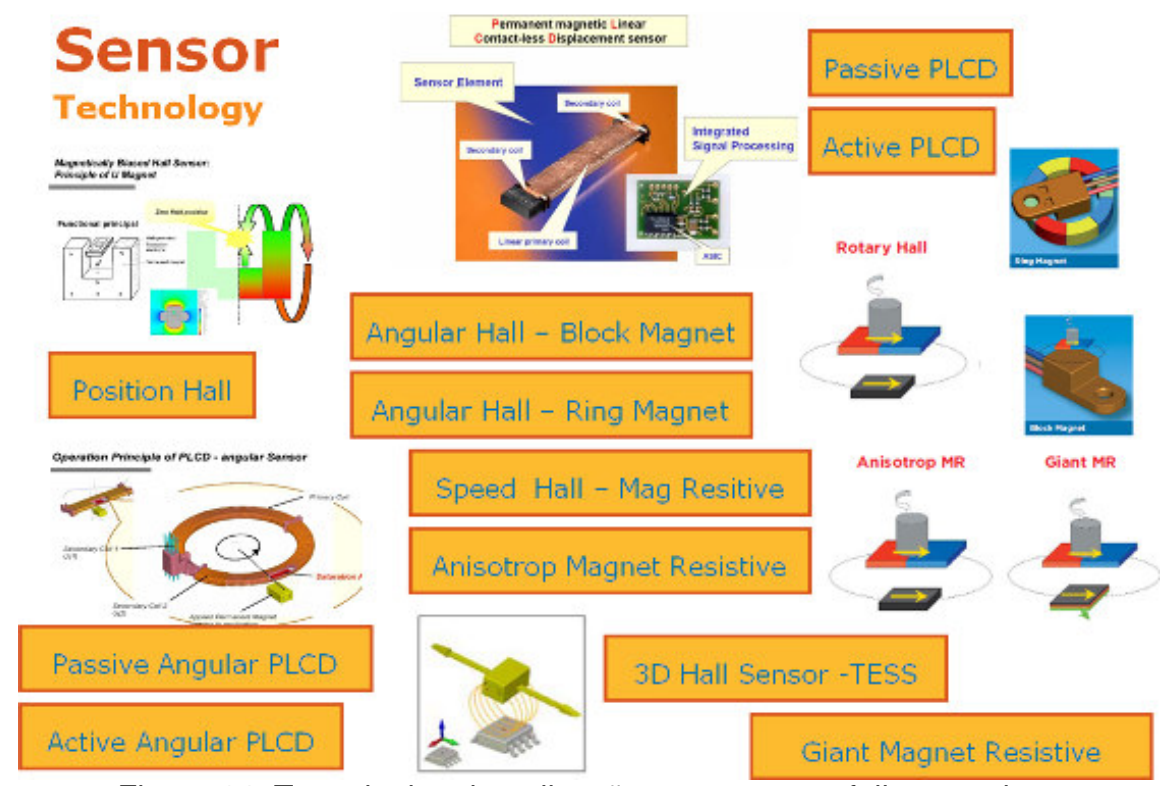

Figura 14: Tecnologias de aplicação em sensores full contactless

A mais tradicionalmente aplicada é demonstrada na figura 15, onde observamos que a incidência de um campo magnético perpendicular a uma estrutura metálica, por onde circula uma corrente, gera uma diferença de potencial que pode ser medida em Volts. A esse fenômeno denomina-se Efeito Hall, que foi descoberto por E.H.Hall em 1879.

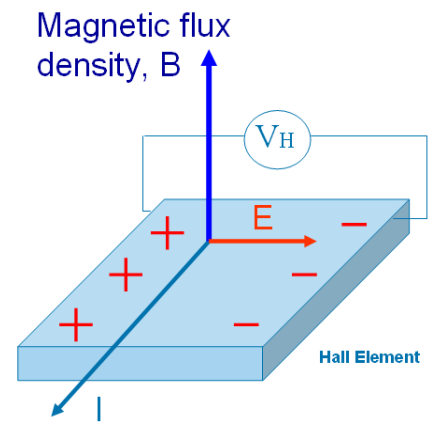

Figura 15: Funcionamento tecnologia Hall

\subsubsection{Sensor 3D Hall (i.e [11])}

A tecnologia de sensoriamento 3D Hall que será aqui detalhada oferece vantagens técnicas e econômicas quando comparada com a tecnologia Hall convencional.

Uma das principais vantagens consiste em tornar possível monitorar deslocamento linear e angular com um único conjunto de elemento sensor e magneto. Sendo que para medir estas duas 
variáveis com a tecnologia Hall convencional seriam necessários dois conjuntos. Outra grande vantagem é que a nova tecnologia permite trabalhar com magnetos de tamanho reduzido, o que ajuda a diminuir o uso de recursos naturais limitados (materiais de terras raras).

De forma geral, podemos dizer que um sensor Hall convencional precisa trabalhar com um magneto cujo tamanho tem que ser da ordem de $60 \%$ do range linear de medição. Ou seja, para medir um deslocamento linear de 30 milímetros seria necessário um magneto de aproximadamente $18 \mathrm{~mm}$ de comprimento. Com a nova tecnologia de Sensor 3D Hall a TE Connectivity reduziu significativamente este requisito, tornado possível, para algumas aplicações, monitorar $30 \mathrm{~mm}$ com um imã de apenas $6 \mathrm{~mm}$ de comprimento. Este grande avanço permite ainda reduzir o tamanho dos sistemas automotivos e aumentar o nível de integração, aumentando ainda mais os potenciais de redução de custos e melhoria da qualidade dos sistemas.

Somado aos benefícios acima, a nova tecnologia de sensor 3D Hall garante melhor linearidade do sinal de saída mesmo em situações críticas para o sistema Hall convencional, ou seja, nos limites extremos do campo de medição do sensor. Isto ocorre porque o a tecnologia de sensor 3D Hall mede dois vetores do campo magnético ( $\mathrm{Y}$ e Z) em vez de medir apenas o fluxo magnético absoluto.

A tecnologia 3D Hall é também mais resistente a vibração, ou seja, viabiliza a sua aplicação em uma gama maior de sistemas cuja aplicação de sensor Hall não era efetiva devido às variações de airgap entre sensor e magneto que ocorrem em conseqüência do nível de vibração do sistema. Esta vantagem também é fruto do princípio vetorial de medição do campo magnético que permite que o air gap entre magneto e sensor seja maior.

A figura 16 representa o grau de liberdade de aplicação da tecnologia de sensoriamento 3D Hall, ou seja, permite monitorar o movimento de elemento magnético nos três eixos (X, Y, Z). Sejam eles movimentos lineares ou angulares.

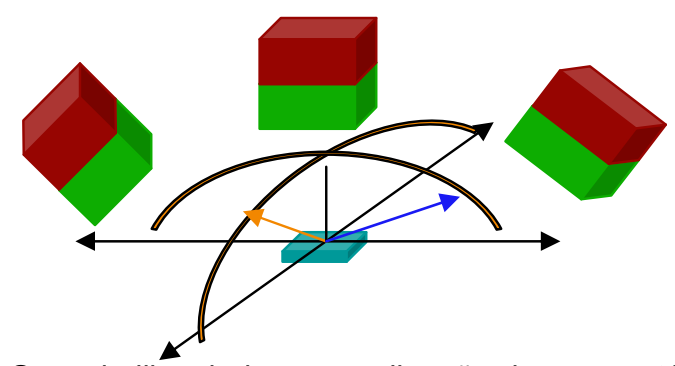

Figura 16: Grau de liberdade para aplicação do sensor 3D Hall 
Para melhor compreensão do princípio de funcionamento da tecnologia de sensor 3D Hall, segue abaixo detalhes técnicos que demonstra a incidência vetorial do campo magnético no elemento sensor.

A grande diferença construtiva entre o sensor Hall clássico e o 3D Hall é que neste caso são utilizados dois elementos sensores independentes sendo um na posição horizontal e outro na posição vertical, desta forma o monitoramento da posição passa a ser consequência da resultante vetorial do campo magnético. Na figura 17 observamos um elemento Hall montado na posição vertical, sendo influenciado pela componente senoidal do campo magnético.

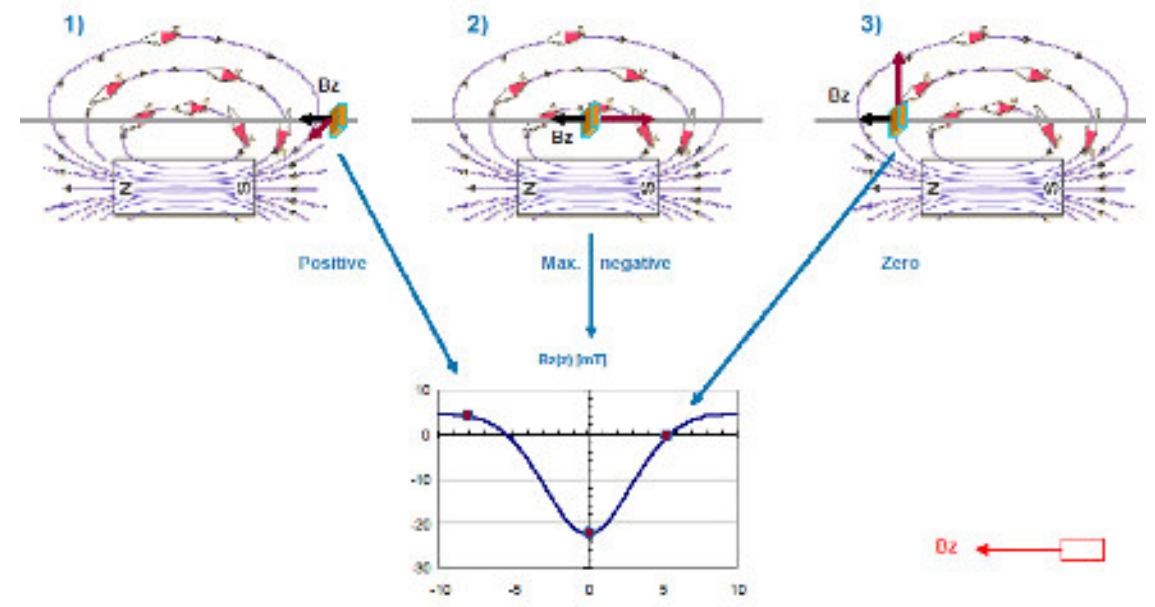

Figura 17: 3D Hall montado na vertical

Na figura 18 observamos um elemento Hall montado na posição horizontal, sendo influenciado pela componente co-senoidal do campo magnético.

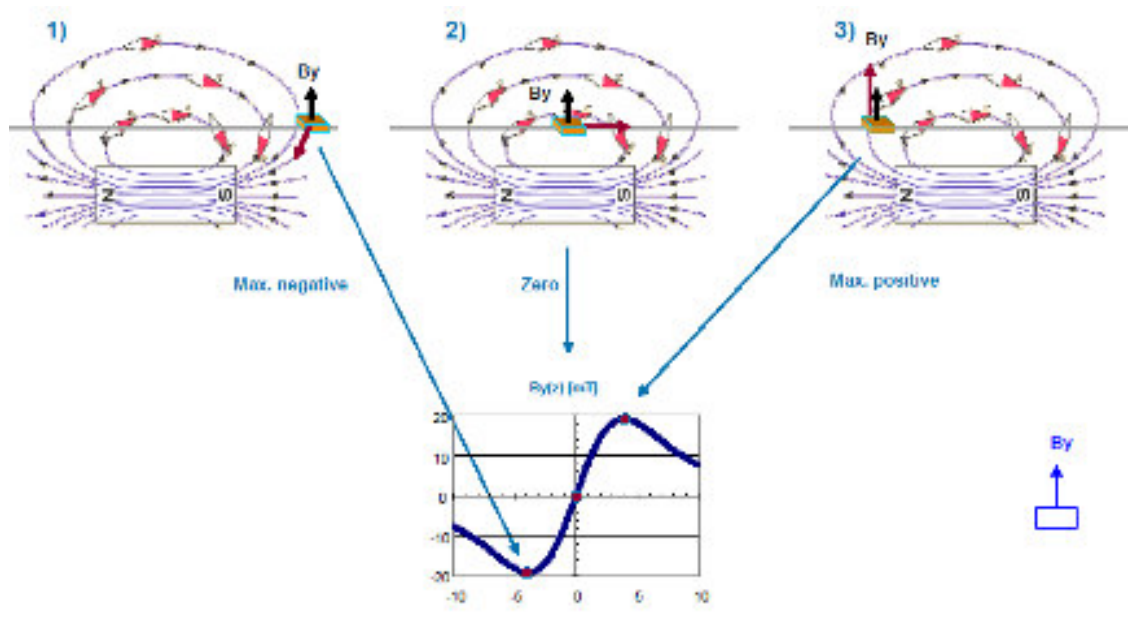

Figura 18: 3D Hall montado na horizontal 
$\mathrm{Na}$ figura 19 observamos que o resultado do monitoramento por meio da tecnologia 3D Hall consiste na resultante entre as leituras do sensor vertical e horizontal (arco tangente).

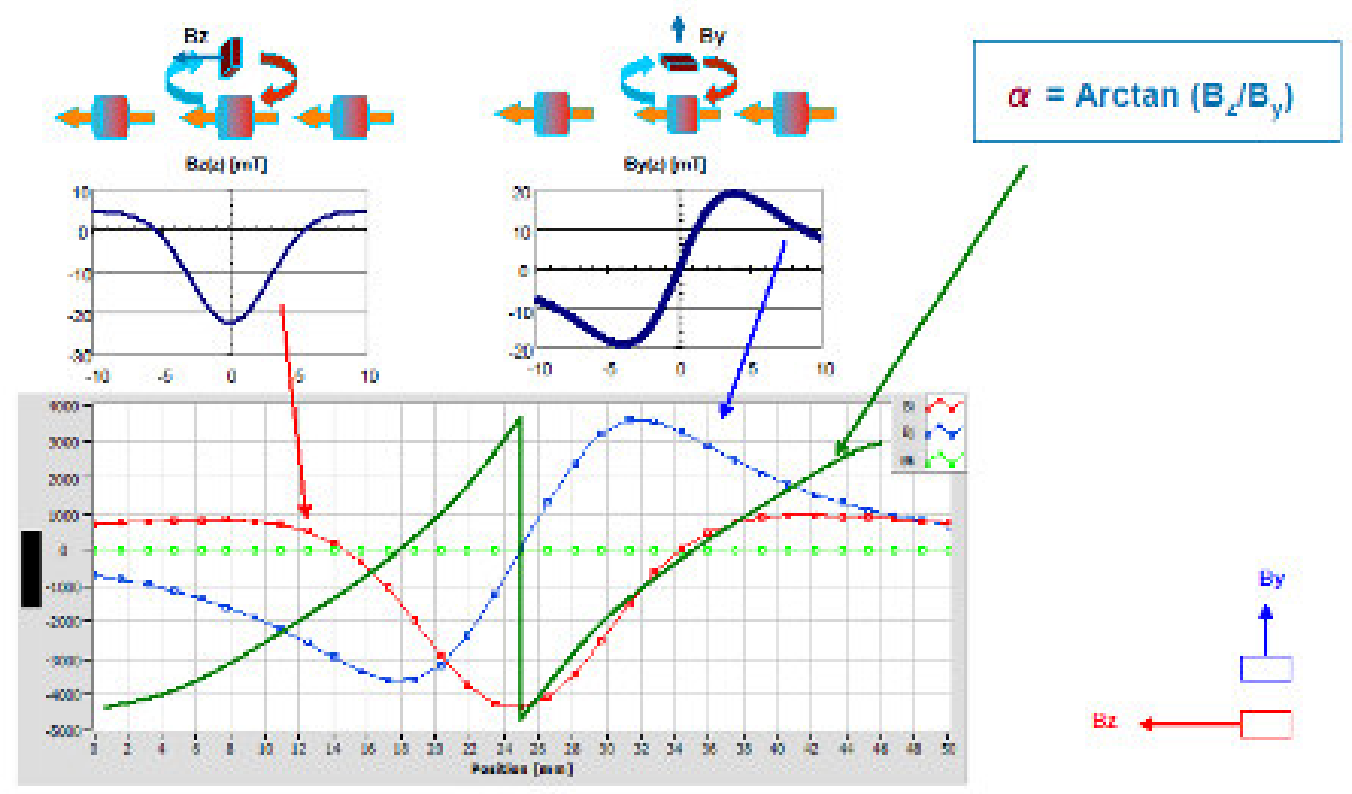

Figura 19: Sinal resultante do 3D Hall

\section{APLICAÇÃO dO SENSOR 3D HALL NO SISTEMA AMT FREE CHOICE®}

Para o estudo de caso neste artigo, utilizaremos o sistema Free Choice $\AA$ classificado como $X Y$, pois os movimentos dos eixos de seleção e engate se assemelham ao movimento ao longo de duas coordenadas (X Y), conforme figura 20.

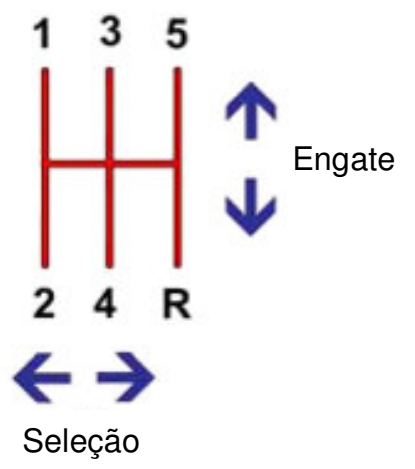

Figura 20: Movimentos X Y

O primeiro eixo, chamado de eixo de seleção, possui três posições possíveis:

- Inferior: seleciona a posição que possibilita o engate da $1^{\text {a }}$ ou $2^{\text {a }}$ marcha;

- Intermediário: seleciona a posição que possibilita 0 engate da $3^{\underline{a}}$ ou $4^{\underline{a}}$ marcha;

- Superior: seleciona a posição que possibilita o engate da $5^{a}$ marcha ou da marcha Ré. 
Duas eletroválvulas do tipo on/off posicionam o eixo de seleção nas posições citadas acima. A figura 21 ilustra o eixo de seleção e suas possíveis posições.

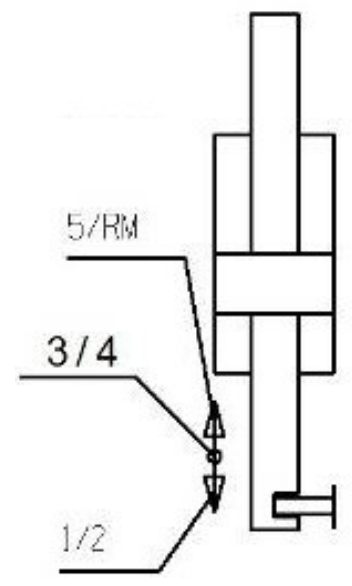

Figura 21: Eixo de seleção

O curso total do eixo de seleção é de $15,15 \mathrm{~mm}$, conforme pode ser visualizado na figura 22.

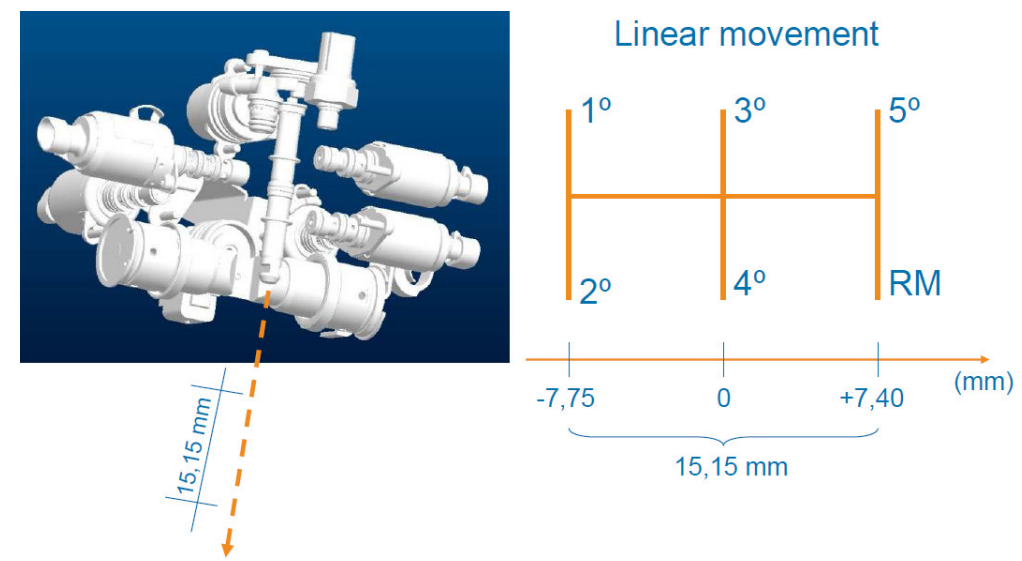

Figura 22: Curso do eixo de seleção

O segundo eixo, chamado de eixo de engate também possui três posições possíveis:

- $\quad$ Par: para engate das marchas pares mais a marcha ré;

- Intermediário: para posição neutra;

- I Ímpar: para engate das marchas ímpares.

Duas válvulas solenóides de pressão posicionam o eixo de engate nas posições citadas acima. A figura 23 ilustra o eixo de engate e suas possíveis posições: 


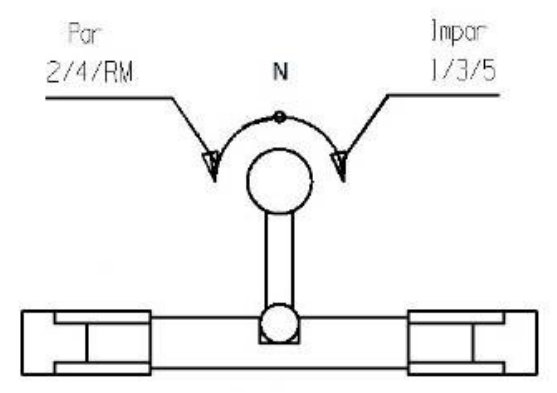

Figura 23:_Eixo de engate

O curso total do eixo de engate é de $27,25^{\circ}$, conforme pode ser visualizado na figura 24.

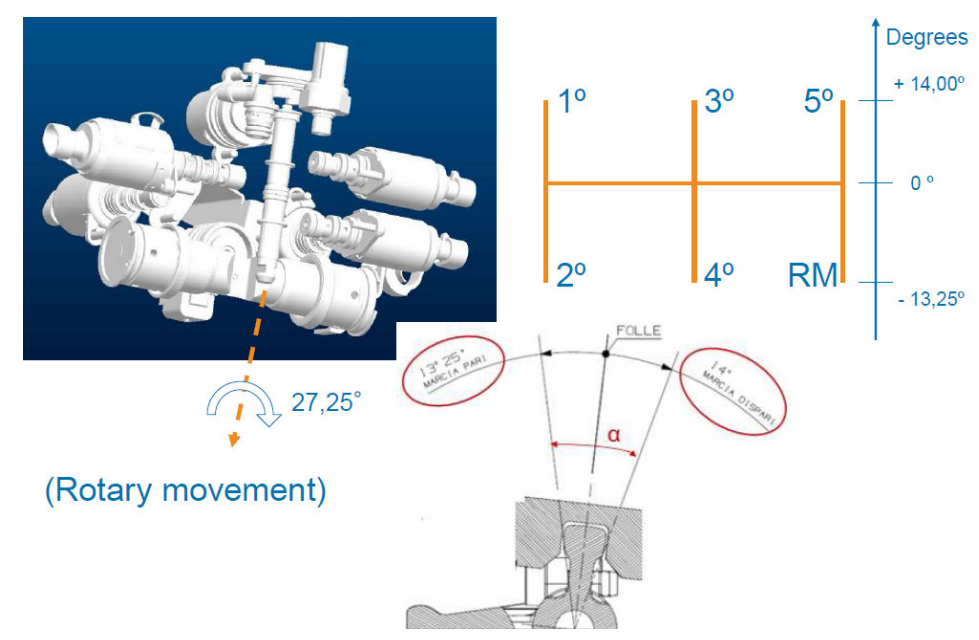

Figura 24: Curso do eixo de engate

A medição dos cursos de seleção e engate são necessárias para que a automação do sistema ocorra em modo "closed loop", portanto nota-se que os itens 3 e 4 da figura 25, que representa o grupo válvulas do Free Choice ${ }^{\circledR}$, são sensores de posição do tipo com contato e são do tipo efeito Hall, sendo um para movimento de seleção e outro para movimento de engate.

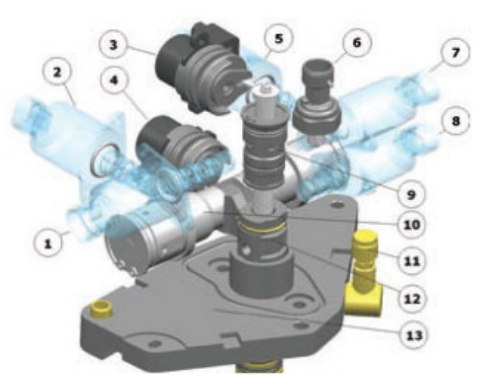

Figura 25: Grupo válvulas do Free Choice® XY: (1) Eletroválvula proporcional de vazão, (2 e 5) Eletroválvula proporcional de pressão, (3) Sensor de posição do atuador de seleção, (4) Sensor de posição do atuador de engate, (6) Sensor de pressão do óleo, (7 e 8) Eletroválvula on/off, (9) Eixo de seleção, (10) Eixo de engate, (11) Respiro, (12) Conector de engate e (13) Flange intermediária 
Devido o sensor ter o contato mecânico, é necessário ainda de um elemento mecânico de ligação, que tem a função de converter o movimento linear do eixo medido (seta azul) em movimento angular do sensor (seta preta), conforme detalhe na figura 26.

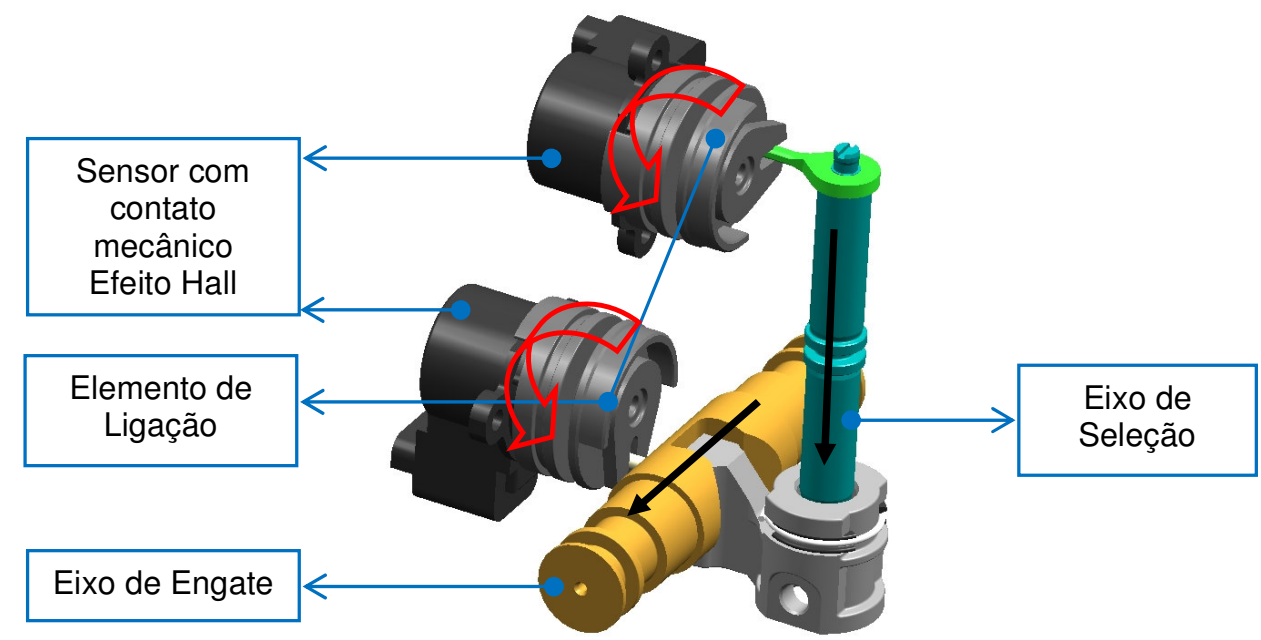

Figura 26: Configuração dos eixos com sensor de contato mecãnico de efeito hall

Para aplicação do sensor com a tecnologia 3D Hall, é necessário a utilização de um magneto montado sobre um componente que desenvolva ambos os movimento de engate e seleção, neste caso no eixo de seleção. Desta forma os dois sensores de efeito Hall e os respectivos elementos de ligação são substituídos por um único sensor 3D Hall e um magneto, conforme demonstrada na figura 27.

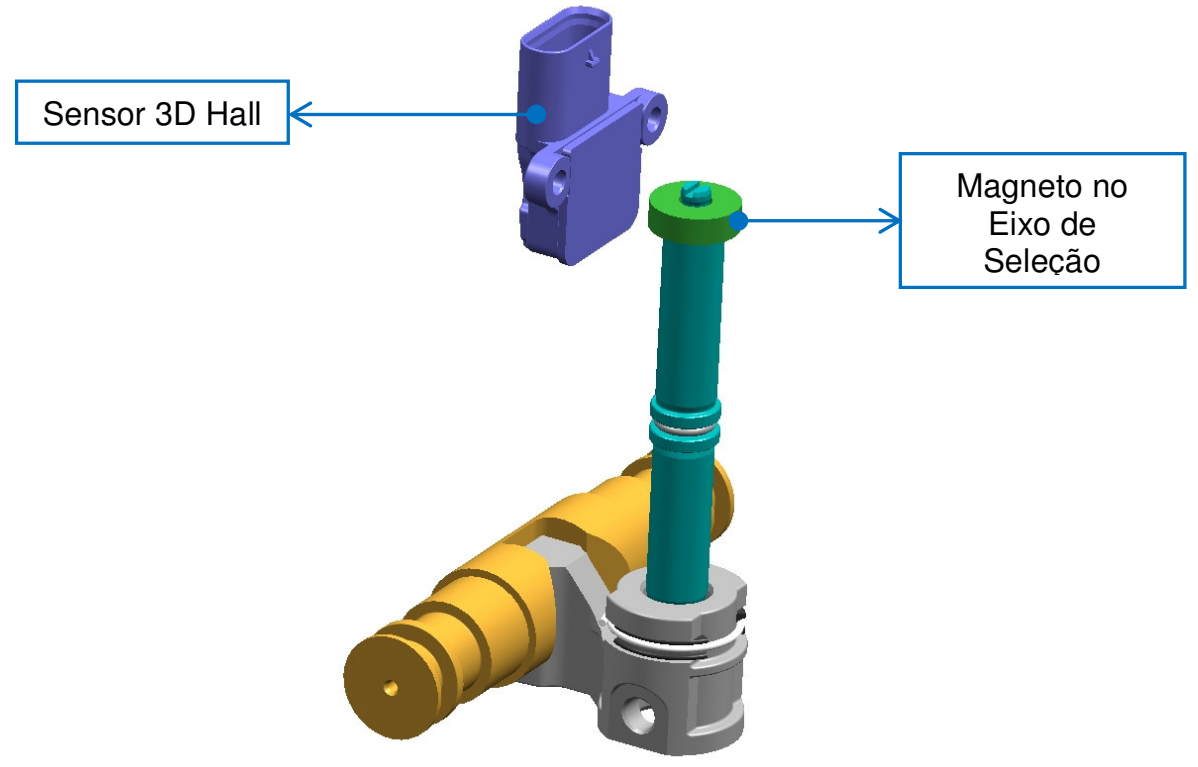

Figura 27: Configuração dos eixos com sensor 3D hall 
$\mathrm{Na}$ figura 28 podemos observar as diferenças proporcionadas ao produto com a utilização do sensor Hall 3D.

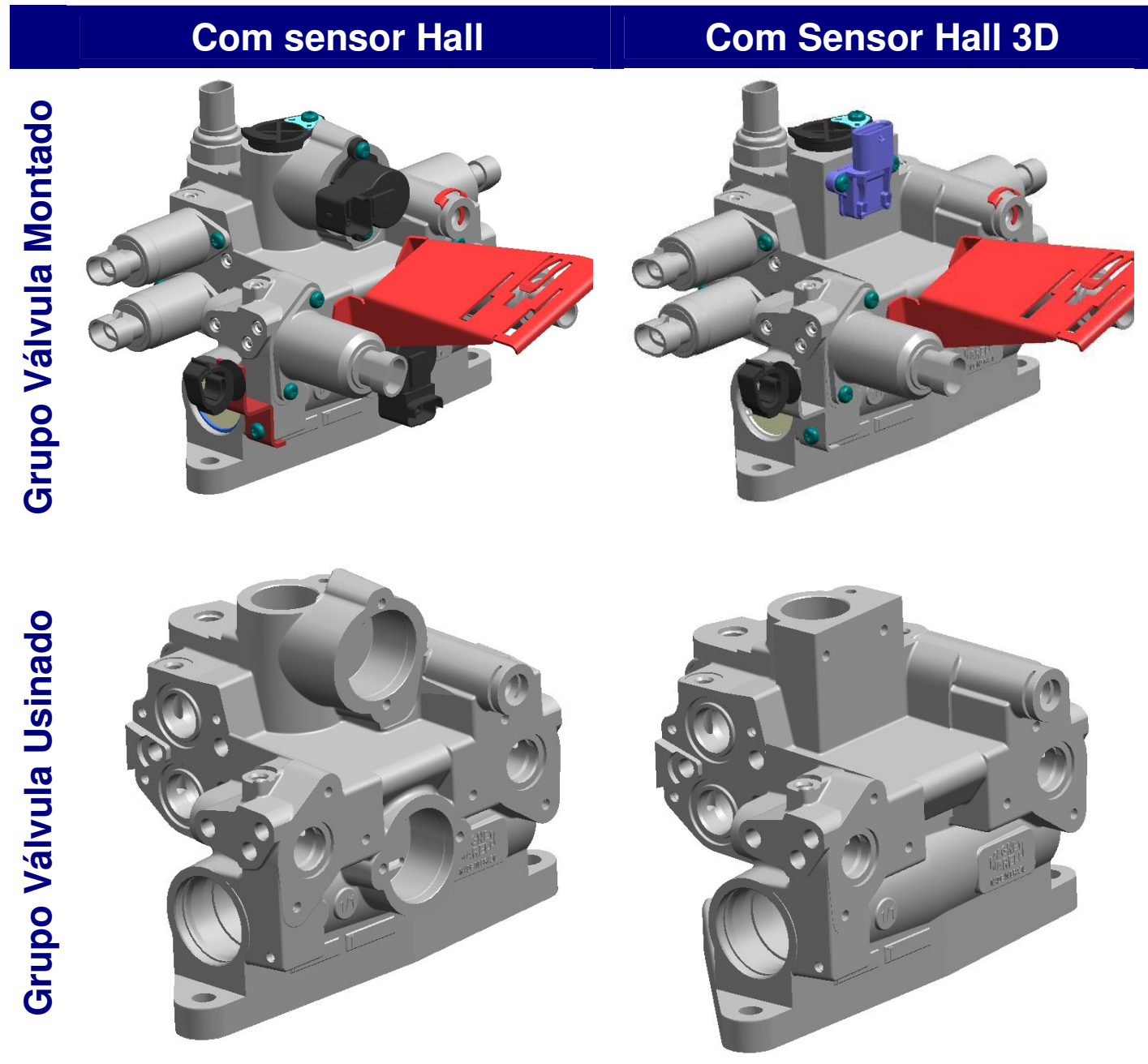

Figura 28: Comparativo produto após implementação sensor Hall 3D

Esta substituição traz ainda as seguintes vantagens técnicas;

- Redução do potencial da falhas em função menor quantidade de componentes e utilização do conceito de sensoriamento "full contactless";

- Redução de tempo de fabricação em função da redução do número de operações no processo produtivo;

- Redução de custo em função da eliminação de componentes;

- Redução de massa do grupo válvula de alumínio em função da eliminação do ponto de montagem de um sensor. 


\section{CONCLUSÃO}

Este artigo evidencia que atualmente no Brasil predomina-se a aplicação de sistema de transmissão manual em função do fator custo. Contudo os fatores abaixo estão fomentando a aplicação de sistemas de transmissão que proporcionam maior nível de conforto e que sejam mais eficientes.

Fatores:

- congestionamento nos grandes centros urbanos;

- $\quad$ aumento dos níveis de emissões de $\mathrm{CO} 2$;

- $\quad$ necessidade de aumento do conteúdo nacional para mitigar a perda de mercado para os veículos importados.

Constatamos também que dentre os sistemas de transmissão, a transmissão AUTOMATIZADA é a que melhor atende as novas necessidades do mercado, com a melhor relação custo benefício.

Constatamos também que para manter o crescimento deste tipo transmissão é importante que o sistema de sensoriamento acompanhe a evolução tecnológica.

Dentre as tecnologias de sensoriamento disponíveis no mercado a tecnologia 3D HALL é a que traz mais vantagens técnicas e também vantagens de custo. A tecnologia 3D Hall é também considerada a tecnologia de vanguarda tecnológica.

Quando a tecnologia 3D Hall é aplicada no sistema de transmissão automatizada Free Choice®, as vantagens se potencializam sendo que os resultados podem ser observados na tabela abaixo:

\begin{tabular}{|c|c|c|c|c|}
\hline Variável & Potenciômetro & $\begin{array}{l}\text { Sem contato } \\
\text { Elétrico }\end{array}$ & $\begin{array}{c}\text { HALL } \\
\text { (Full } \\
\text { Contactless) }\end{array}$ & $\begin{array}{c}\text { 3D HALL } \\
\text { (Full } \\
\text { Contactless) }\end{array}$ \\
\hline $\begin{array}{l}\text { Quantidade de } \\
\text { sensores }\end{array}$ & 2 & 2 & 2 & 1 \\
\hline $\begin{array}{l}\text { Expectativa de } \\
\text { vida }\end{array}$ & & & & \\
\hline $\begin{array}{l}\text { Redução de } \\
\text { Custo }\end{array}$ & Ref. & & & \\
\hline $\begin{array}{l}\text { Pontos de } \\
\text { Fixação }\end{array}$ & 4 & 4 & 4 & 2 \\
\hline Conectores & 2 & 2 & 2 & 1 \\
\hline $\begin{array}{l}\text { Redução de } \\
\text { Massa }\end{array}$ & Ref. & 0 & 0 & $1 \%$ \\
\hline
\end{tabular}


Com isto concluímos que a tecnologia de sensoriamento 3D Hall aplicada em sistema eletrônico de comando automatizado da transmissão é uma solução que realmente coloca o Brasil na vanguarda tecnológica e atende aos principais metas do regime automotivo Inovar Auto.

\section{REFERÊNCIAS}

[1] VEJA, Abril editora. O impacto do caos nas ruas. Disponível em http://veja.abril.com.br/idade/exclusivo/transito/contexto1.html. Acesso em 20 de maio de 2013.

[2] ORTIZ Diego. Poupe seu pé esquerdo. O Estado de São Paulo - Jornal do Carro, São Paulo, Ano134, 8JC, 10 de abril de 2013.

[3] CESVI Brasil - Centro de Experimentação e Segurança Viária. Disponível em http://falatorioautomotivo.blogspot.com/2013/01/cesvi-brasil-explica-os-sistemasde.html. Acesso em 15 de maio de 2013.

[4] Sistemas automotivos: Transmissão. Disponível em http://sistemasautomotivos.blogspot.com/2009/01/transmisso.html. A acesso em 15 de maio de 2013

[5] DAL POGGOTTO, Priscila. Notícia G1. Disponível em http://g1.globo.com/carros/noticia/2012/12/cambio-cvt-e-nova-tendencia-nos-carrosbrasileiros-apos-novas-regras.html. Acesso em 15 de maio de 2013.

[6] Blog Auto Esporte. Revista Auto esporte. Diposnível em http://colunas.revistaautoesporte.globo.com/blogdaautoesporte/2011/05/20/qualcomprar-tipos-de-cambio-automatico/. Acesso em 15 de maio de 2013.

[7] Sensores Automotivos. TE Connectivity. Disponível em http://www.te.com/en/industries/automotive/browse-products/sensors/transmissionsensors.html. Acesso em 15 de maio de 2013.

[8] Sensores Automotivos, TE Connectivity. Disponível em http://www.te.com/automotive/pdf/1308086-2.pdf. Acesso em 15 de maio de 2013.

[9] Tecnologia sensor com contato. Disponível em http://en.wikipedia.org/wiki/Potentiometer. Acesso em 15 de maio de 2013.

[10] Tecnologia sensor Hall. Disponível em http://en.wikipedia.org/wiki/Hall effect sensor. Acesso em 15 de maio de 2013.

[11] Tecnologia sensor 3D Hall. Disponível em http://www.te.com/en/aboutte/news/new-products/2012/05/09/new-3d-hall-sensor-series-for-automotiveapplications.html. Acesso em 15 de maio de 2013. 
NAUNHEIMER, Harald; LECHNER, Gisbert.. Automotive Transmissions Fundamentals, Selection, Design and Application. Alemanha:Springer, 2011. 